\title{
Effects of Hall Current and Ion-Slip on Unsteady MHD Couette Flow
}

\author{
Nirmal Ghara, Sovan Lal Maji, Sanatan Das, Rabindranath Jana', Swapan Kumar Ghosh ${ }^{2}$ \\ ${ }^{1}$ Department of Applied Mathematics, Vidyasagar University, Midnapore, India \\ ${ }^{2}$ Department of Mathematics, Narajole Raj College, Narajole, India \\ Email: jana261171@yahoo.co.in,g_swapan2002@yahoo.com
}

Received November 26, 2011; revised December 15, 2011; accepted December 28, 2011

\begin{abstract}
The unsteady MHD Couette flow of an incompressible viscous electrically conducting fluid between two infinite nonconducting horizontal porous plates under the boundary layer approximations has been studied with the consideration of both Hall currents and ion-slip. An analytical solution of the governing equations describing the flow is obtained by the Laplace transform method. It is seen that the primary velocity decreases while the magnitude of secondary velocity increases with increase in Hall parameter. It is also seen that both the primary velocity and the magnitude of secondary velocity decrease with increase in ion-slip parameter. It is observed that a thin boundary layer is formed near the stationary plate for large values of squared Hartmann number and Reynolds number. The thickness of this boundary layer increases with increase in either Hall parameter or ion-slip parameter.
\end{abstract}

Keywords: MHD; Couette Flow; Porous Plate; Hall Current; Ion-Slip; Hartmann Number and Reynolds Number

\section{Introduction}

The magnetohydrodynamic (MHD) flow between two parallel plates, one in uniform motion and the other held at rest known as MHD Couette flow, is a classical problem that has many applications in MHD power generators and pumps, accelerators, aerodynamic heating, electrostatic precipitation, polymer technology, petroleum industry, purification of crude oil and fluid droplets and sprays. A lot of research work concerning the MHD Couette flow has been obtained under different physical effects. In most cases, the Hall and ion slip terms were ignored in applying Ohm's law as they have no marked effect for small and moderate values of the magnetic field. However, the current trend for the application of magnetohydrodynamics is towards a strong magnetic field, so that the influence of electromagnetic force is noticeable [1]. Under these conditions, the Hall currents and ion slip are important and they have a marked effect on the magnitude and direction of the current density and consequently on the magnetic force term. Soundalgekar [2] has studied the Hall and ion-slip effects in MHD Coutte flow with heat transfer. Attia [3] has studied the unsteady Couette flow with heat transfer considering ionslip. The transient Hartmann flow with heat transfer considering the ion slip has been investigated by Attia [4-6]. Attia [7] has obtained the analytical solution for flow of a dusty fluid in a circular pipe with Hall and ion slip effect.
Seddeek [8] has studied the effects of Hall and ion-slip currents on magneto-micropolar fluid and heat transfer over a non-isothermal stretching sheet with suction and blowing. The effects of Hall and ion-slip currents on free convective heat generating flow in a rotating fluid have studied by Ram [9]. Mittal and Bhat [10] has discussed the forced convective heat transfer in a MHD channel with Hall and ion slip currents. Jana and Datta [11] has described the Couette flow and heat transfer in a rotating system. The Hall effect on unsteady Couette flow under boundary layer approximations has been analysed by Kanch and Jana [12]. Attia [13] has studied the ion slip effect on unsteady Couette flow with heat transfer under exponential decaying pressure gradient. The combined effect of Hall and ion-slip currents on unsteady MHD Couette flows in a rotating system have been investigated by Jha and Apere [14].

The present paper is devoted to study the combined effects of Hall current and ion-slip on the unsteady MHD Couette flow between two infinite horizontal parallel porous plates under the boundary layer approximations. The upper plate is moving with a uniform velocity $U$ while the lower plate is held at rest. The fluid is acted upon by a constant pressure gradient and a uniform suction/injection at the plates. A uniform magnetic field $B_{0}$ is applied perpendicular to the plates. It is found that the primary velocity decreases while the magnitude of the secondary velocity increases with increase in Hall pa- 
rameter. It also is found that both the primary velocity and the magnitude of secondary velocity decrease with increase in ion-slip parameter. Asymptotic behavior of the solution has been analyzed for large values of squared Hartmann number and Reynolds number. It is observed that a thin boundary layer is formed near the stationary plate for large values of the magnetic parameter and Reynolds number. The thickness of this boundary layer increases with increases in either Hall parameter or ion-slip parameter. Further, it is seen that the shear stress components $\tau_{x_{0}}$ and $\tau_{z_{0}}$ due to the primary and secondary flows at the stationary plate $\eta=0$ increase with increase in Hall parameter for fixed value of squared Hartmann number and ion slip parameter. It is also seen that for fixed value of both squared Hartmann number and Hall parameter, $\tau_{x_{0}}$ increases while $\tau_{z_{0}}$ decreases with increase in ion-slip parameter.

\section{Mathematical Formulation and Its Solution}

Consider the viscous incompressible electrically conducting fluid bounded by two infinite horizontal parallel porous plates separated by a distance $h$. Choose a Cartesian co-ordinate system with $x$-axis along the lower stationary plate in the direction of the flow, the $y$-axis is normal to the plates and the $z$-axis perpendicular to $x y$ plane (see Figure 1). Initially, at time $t=0$, both the plates are at rest. At time $t>0$, the upper plate suddenly starts to move with uniform velocity $U$ along $x$-axis. A uniform magnetic field $B_{0}$ is applied perpendicular to the plates. The velocity components are $(u, v, w)$ relative to a frame of reference. Since the plates are infinitely long, all physical variables, except pressure, depend on $y$ and $t$ only. The equation of continuity then gives

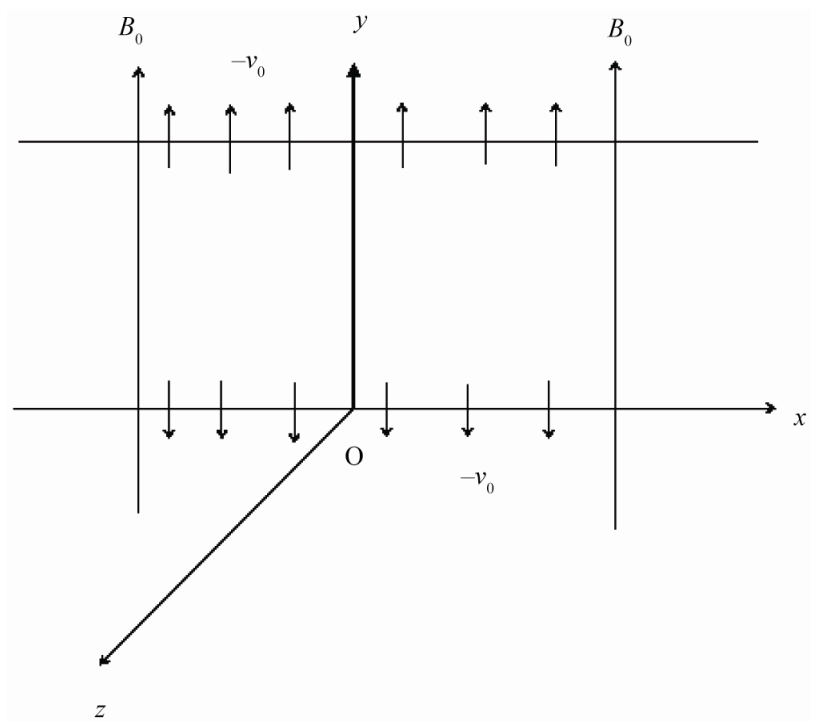

Figure 1. Geometry of the problem. $v=-v_{0}$ everywhere in the flow where $v_{0}$ is the suction velocity at the plates.

Taking the effects of ion-slip, the generalised Ohm's law is

$$
\boldsymbol{j}=\sigma(\boldsymbol{E}+\boldsymbol{q} \times \boldsymbol{B})-\frac{\omega_{e} \tau_{e}}{B_{0}}(\boldsymbol{j} \times \boldsymbol{B})-\frac{\omega_{e} \tau_{e} \beta_{i}}{B_{0}^{2}}[(\boldsymbol{j} \times \boldsymbol{B}) \times \boldsymbol{B}],
$$

where $\boldsymbol{B}, \boldsymbol{E}, \boldsymbol{q}, \boldsymbol{j}, \sigma, \omega_{e}, \tau_{e}$ and $\beta_{i}$ are respectively, the magnetic field vector, the electric field vector, the fluid velocity vector, the current density vector, the conductivity of the fluid, the cyclotron frequency, the electron collision time and $\beta_{i}$ the ion-slip parameter.

We shall assume that the magnetic Reynolds number for the flow is small so that the induced magnetic field can be neglected. This assumption is justified since the magnetic Reynolds number is generally very small for partially ionized gases. The solenoidal relation $\nabla \cdot \boldsymbol{B}=0$ for the magnetic field gives $B_{y}=B_{0}=$ constant everywhere in the flow where $\boldsymbol{B} \equiv\left(B_{x}, B_{y}, B_{z}\right)$. The equation of the conservation of the charge $\nabla \cdot \boldsymbol{j}=0$ gives $j_{y}=$ constant. This constant is zero since $j_{y}=0$ at each plate which is electrically non-conducting. Thus $j_{y}=0$ everywhere in the flow. Since the induced magnetic fields are neglected, the Maxwel's equation $\nabla \times \boldsymbol{E}=-\frac{\partial \boldsymbol{B}}{\partial t}$ becomes $\nabla \times \boldsymbol{E}=0$ which gives $\frac{\partial E_{x}}{\partial z}=0$ and $\frac{\partial E_{z}}{\partial z}=0$. This implies that $E_{x}=$ constant and $E_{z}=$ constant everywhere in the flow.

In view of the above assumption, Equation (1) gives

$$
\begin{aligned}
& \left(1+\beta_{e} \beta_{i}\right) j_{x}-\beta_{e} j_{z}=E_{x}-\sigma B_{0} w, \\
& \left(1+\beta_{e} \beta_{i}\right) j_{z}+\beta_{e} j_{x}=E_{z}+\sigma B_{0} u,
\end{aligned}
$$

where $\beta_{e}=\omega_{e} \tau_{e}$ is the Hall parameter. Solving for $j_{x}$ and $j_{z}$, we get

$$
\begin{aligned}
j_{x}= & \frac{\sigma}{\left(1+\beta_{e} \beta_{i}\right)^{2}+\beta_{e}^{2}} \\
& \times\left[\left(1+\beta_{e} \beta_{i}\right)\left(E_{x}-B_{0} w\right)+\beta_{e}\left(E_{z}+B_{0} u\right)\right], \\
j_{z}= & \frac{\sigma}{\left(1+\beta_{e} \beta_{i}\right)^{2}+\beta_{e}^{2}} \\
& \times\left[\left(1+\beta_{e} \beta_{i}\right)\left(E_{z}+B_{0} u\right)-\beta_{e}\left(E_{x}-B_{0} w\right)\right] .
\end{aligned}
$$

On the use of Equations (4) and (5), the equations of motion along $x$ - and $y$-directions are

$$
\begin{aligned}
\frac{\partial u}{\partial t}-v_{0} \frac{\partial u}{\partial y}= & -\frac{1}{\rho} \frac{\partial p}{\partial x}+v \frac{\partial^{2} u}{\partial y^{2}}-\frac{\sigma B_{0}}{\rho\left[\left(1+\beta_{e} \beta_{i}\right)^{2}+\beta_{e}^{2}\right]} \\
& {\left[\left(1+\beta_{e} \beta_{i}\right)\left(E_{z}+B_{0} u\right)-\beta_{e}\left(E_{x}-B_{0} w\right)\right] }
\end{aligned}
$$




$$
\begin{gathered}
0=-\frac{1}{\rho} \frac{\partial p}{\partial y} \\
\frac{\partial w}{\partial t}-v_{0} \frac{\partial w}{\partial y}=-\frac{1}{\rho} \frac{\partial p}{\partial z}+v \frac{\partial^{2} w}{\partial y^{2}}-\frac{\sigma B_{0}}{\rho\left[\left(1+\beta_{e} \beta_{i}\right)^{2}+\beta_{e}^{2}\right]} \\
\times\left[\left(1+\beta_{e} \beta_{i}\right)\left(E_{x}-B_{0} w\right)+\beta_{e}\left(E_{z}+B_{0} u\right)\right]
\end{gathered}
$$

where $\rho, v$ and $p$ are respectively the fluid density, the kinematic coefficient of viscosity and the fluid pressure.

The boundary conditions are

$$
\begin{array}{lll}
u=w=0 & \text { for } t \leq 0 & \text { for all } y, \\
u=w=0 & \text { at } y=0 & \text { for } t>0, \\
u=U, w=0 & \text { at } y=h & \text { for } t>0 .
\end{array}
$$

Equation (7) shows the constancy of pressure along $y$-axis. Also, the fluid flow within the channel is induced due to uniform motion of the upper plate $y=h$. Therefore, using boundary condition at $y=h$ in Equations (6) and (8) we get

$$
\begin{aligned}
0= & -\frac{1}{\rho} \frac{\partial p}{\partial x}-\frac{\sigma B_{0}}{\rho\left[\left(1+\beta_{e} \beta_{i}\right)^{2}+\beta_{e}^{2}\right]} \\
& \times\left[\left(1+\beta_{e} \beta_{i}\right)\left(E_{z}+B_{0} U\right)-\beta_{e} E_{x}\right], \\
0= & -\frac{1}{\rho} \frac{\partial p}{\partial z}-\frac{\sigma B_{0}}{\rho\left[\left(1+\beta_{e} \beta_{i}\right)^{2}+\beta_{e}^{2}\right]} \\
& \times\left[\left(1+\beta_{e} \beta_{i}\right) E_{x}+\beta_{e}\left(E_{z}+B_{0} U\right)\right] .
\end{aligned}
$$

On the use of (10) and (11), Equations (6) and (8), under the usual boundary layer approximations become

$$
\begin{aligned}
\frac{\partial u}{\partial t}-v_{0} \frac{\partial u}{\partial y}= & v \frac{\partial^{2} u}{\partial y^{2}}-\frac{\sigma B_{0}^{2}}{\rho\left[\left(1+\beta_{e} \beta_{i}\right)^{2}+\beta_{e}^{2}\right]} \\
& \times\left[\left(1+\beta_{e} \beta_{i}\right)(u-U)+\beta_{e} w\right], \\
\frac{\partial w}{\partial t}-v_{0} \frac{\partial w}{\partial y} & =v \frac{\partial^{2} w}{\partial y^{2}}-\frac{\sigma B_{0}^{2}}{\rho\left[\left(1+\beta_{e} \beta_{i}\right)^{2}+\beta_{e}^{2}\right]} \\
& \times\left[\left(1+\beta_{e} \beta_{i}\right) w-\beta_{e}(u-U)\right],
\end{aligned}
$$

Introducing the non-dimensional variables

$$
\eta=\frac{y}{h}, \quad u_{1}=\frac{u}{U}, \quad w_{1}=\frac{w}{U}, \tau=\frac{v t}{h^{2}},
$$

Equations (12) and (13) become

$$
\bar{F}(\eta, s)=\frac{e^{-\frac{\mathrm{Re}}{2} \eta}}{s}\left[\cosh \left\{\left(\frac{\mathrm{Re}^{2}}{4}+a\right)+s\right\}^{\frac{1}{2}} \eta-\frac{\cosh \left\{\left(\frac{\mathrm{Re}^{2}}{4}+a\right)+s\right\}^{\frac{1}{2}}}{\sinh \left\{\left(\frac{\mathrm{Re}^{2}}{4}+a\right)+s\right\}^{\frac{1}{2}}} \sinh \left\{\left(\frac{\mathrm{Re}^{2}}{4}+a\right)+s\right\}^{\frac{1}{2}} \eta\right] .
$$

$$
\begin{aligned}
\frac{\partial u_{1}}{\partial \tau}-\operatorname{Re} \frac{\partial u_{1}}{\partial \eta}= & \frac{\partial^{2} u_{1}}{\partial \eta^{2}}-\frac{M^{2}}{\left[\left(1+\beta_{e} \beta_{i}\right)^{2}+\beta_{e}^{2}\right]} \\
& \times\left[\left(1+\beta_{e} \beta_{i}\right)\left(u_{1}-1\right)+\beta_{e} w_{1}\right], \\
\frac{\partial w_{1}}{\partial \tau}-\operatorname{Re} \frac{\partial w_{1}}{\partial \eta}= & \frac{\partial^{2} w_{1}}{\partial \eta^{2}}-\frac{M^{2}}{\left[\left(1+\beta_{e} \beta_{i}\right)^{2}+\beta_{e}^{2}\right]} \\
& \times\left[\left(1+\beta_{e} \beta_{i}\right) w_{1}-\beta_{e}\left(u_{1}-1\right)\right],
\end{aligned}
$$

where $M=\left[\left(\frac{\sigma}{\rho v}\right)^{\frac{1}{2}} B_{0} h\right]$ is the Hartmann number and $\operatorname{Re}=\frac{v_{0} U}{v}$ the Reynolds number.

Equations (15) and (16) can be combined into the following equation

$$
\frac{\partial F}{\partial \tau}-\operatorname{Re} \frac{\partial F}{\partial \eta}=\frac{\partial^{2} F}{\partial \eta^{2}}-\left[\frac{M^{2}\left\{\left(1+\beta_{e} \beta_{i}\right)-i \beta_{e}\right\}}{\left(1+\beta_{e} \beta_{i}\right)^{2}+\beta_{e}^{2}}\right] F,
$$

where

$$
F=u_{1}+i w_{1}-1, \quad i=\sqrt{-1} .
$$

The initial and boundary conditions for $F(\eta, \tau)$ are

$$
\begin{array}{ll}
F(\eta, 0)=0 & \text { for all } \eta, \\
F(0, \tau)=-1 \text { and } F(0, \tau)=0 & \text { for } \tau>0
\end{array}
$$

Taking Laplace transform, Equation (17) becomes

$$
\frac{\mathrm{d}^{2} \bar{F}}{\mathrm{~d} \eta^{2}}+\operatorname{Re} \frac{\mathrm{d} \bar{F}}{\mathrm{~d} \eta}-(a+s) \bar{F}=0,
$$

where

$$
\bar{F}(\eta, s)=\int_{0}^{\infty} e^{-s \tau} F(\eta, \tau) \mathrm{d} \tau
$$

and

$$
a=\frac{M^{2}\left\{\left(1+\beta_{e} \beta_{i}\right)-i \beta_{e}\right\}}{\left(1+\beta_{e} \beta_{i}\right)^{2}+\beta_{e}^{2}} .
$$

The boundary conditions (19) become

$$
\bar{F}(0, s)=-\frac{1}{s} \text { and } \bar{F}(1, s)=0 .
$$

The solution of the Equation (20) subject to the boundary condition (23) is 
Taking inverse transform of (24) and on using (18), we get (see Equation (25))

where

$$
\begin{aligned}
& \alpha_{1}=\frac{1}{\sqrt{2}}\left[\left\{\left(\frac{\mathrm{Re}^{2}}{4}+\alpha\right)^{2}+\beta^{2}\right\}^{\frac{1}{2}}+\left(\frac{\mathrm{Re}^{2}}{4}+\alpha\right)\right]^{\frac{1}{2}} \\
& \beta_{1}=\frac{1}{\sqrt{2}}\left[\left\{\left(\frac{\mathrm{Re}^{2}}{4}+\alpha\right)^{2}+\beta^{2}\right\}^{\frac{1}{2}}-\left(\frac{\mathrm{Re}^{2}}{4}+\alpha\right)\right]^{\frac{1}{2}} \\
& \alpha=\frac{M^{2}\left(1+\beta_{e} \beta_{i}\right)}{\left(1+\beta_{e} \beta_{i}\right)^{2}+\beta_{e}^{2}}, \quad \beta=\frac{M^{2} \beta_{e}}{\left(1+\beta_{e} \beta_{i}\right)^{2}+\beta_{e}^{2}} \\
& s=-n^{2} \pi^{2}-\left(\alpha_{1}-\beta_{1}\right)^{2} .
\end{aligned}
$$

On separating into real and imaginary parts one can easily obtain the velocity components $u_{1}$ and $w_{1}$ from Equation (25). The solution given by (25) exists for both $\operatorname{Re}<0$ (corresponding to $v_{0}<0$, for the blowing at the plates) and $\operatorname{Re}>0$ (corresponding to $v_{0}>0$, for the suction at the plates).

\section{Solution at Small Times}

In this case, method used by Carslaw and Jaegar [15] is used since it converges rapidly for small times. For small time $\tau$ which correspond to large $s$, Equation (24) becomes

$$
\bar{F}(\eta, s)=-e^{-\frac{1}{2} \operatorname{Re} \eta} \sum_{m=0}^{\infty} \frac{1}{S}\left[e^{-(2 m+\eta) r}-e^{-(2 m+2-\eta) r}\right]
$$

The inverse transform of (27) is

$$
F(\eta, \tau)=-e^{-\frac{\mathrm{Re}}{2} \eta-\frac{\mathrm{Re}^{2}}{4} \tau-a \tau} \times \sum_{m=0}^{\infty}\left(\frac{\mathrm{Re}^{2}}{4}+a\right)^{n}(4 \tau)^{n} i^{2 n} T_{2 n},
$$

where $i^{n} \operatorname{erfc}(x)$ denotes the repeated integrals of the complementary error function given by

$$
\begin{aligned}
& i^{n} \operatorname{erfc}(x)=\int_{x}^{\infty} i^{n-1} \operatorname{erfc}(\xi) \mathrm{d} \xi, n=0,1,2, \cdots, \\
& i^{0} \operatorname{erfc}(x)=\operatorname{erfc}(x), \\
& i^{-1} \operatorname{erfc}(x)=\frac{2}{\sqrt{\pi}} e^{-x^{2}} .
\end{aligned}
$$

On the use of (18), we have

$$
u_{1}=1-e^{-\left(\frac{\mathrm{Re}}{2} \eta+\alpha^{*} \tau\right)} \times\left[A(\eta, \tau) \cos \beta^{*} \tau+B(\eta, \tau) \sin \beta^{*} \tau\right] \text {, }
$$

$$
w_{1}=-e^{-\left(\frac{\mathrm{Re}}{2} \eta+\beta^{*} \tau\right)} \times\left[A(\eta, \tau) \sin \beta^{*} \tau-B(\eta, \tau) \cos \beta^{*} \tau\right],
$$

where

$$
\begin{aligned}
A(\eta, \tau)= & T_{0}+\alpha \times(4 \tau) T_{2}+\left(\alpha^{*_{2}}-\beta^{*_{2}}\right)(4 \tau)^{2} T_{4} \\
& +\left(\alpha^{* 3}-3 \alpha^{*} \beta^{*_{2}}\right)(4 \tau)^{3} T_{6} \\
& +\left(\alpha^{* 4}-6 \alpha^{*_{2}} \beta^{*_{2}}+\beta^{* 4}\right)(4 \tau)^{4} T_{8}+\cdots \\
B(\eta, \tau)= & \beta^{*}(4 \tau) T_{2}+2 \alpha^{*} \beta^{*}(4 \tau)^{2} T_{4} \\
& +\left(\beta^{* 3}-3 \alpha^{* 2} \beta^{*}\right)(4 \tau)^{3} T_{6} \\
& +4 \alpha^{*} \beta^{*}\left(\alpha^{* 2}-\beta^{* 2}\right)(4 \tau)^{4} T_{8}+\cdots, \\
\alpha^{*}=\frac{\operatorname{Re}^{2}}{4} & +\alpha, \quad \beta^{*}=\beta
\end{aligned}
$$

where $\alpha$ and $\beta$ are given by (26) with

$$
\begin{aligned}
& T_{2 n}=\sum_{m=0}^{\infty}\left[i^{2 n} \operatorname{erfc}\left(\frac{2 m+\eta}{2 \sqrt{\tau}}\right)-i^{2 n} \operatorname{erfc}\left(\frac{2 m+2-\eta}{2 \sqrt{\tau}}\right)\right], \\
& n=0,1,2,3, \cdots
\end{aligned}
$$

Equations (30) and (31) show that the Hall effects become important only when terms of order $\tau$ is taken into account.

\section{Results and Discussion}

To study the effects of suction/blowing, Hall parameter, ion-slip parameter and time on the velocity distributions we have presented the non-dimensional velocity components $u_{1}$ and $w_{1}$ against $\eta$ in Figures 2-6 for various values of the squared-Hartmann number $M^{2}$, Reynolds number $\operatorname{Re}$, Hall parameter $\beta_{e}$, ion-slip parameter $\beta_{i}$ and time $\tau$. It is seen from Figure 2 that both the primary velocity $u_{1}$ and the magnitude of secondary velocity $w_{1}$ increase with increase in $M^{2}$. Figure 3 reveals that the primary velocity $u_{1}$ increases whereas the magnitude of secondary velocity $w_{1}$ decreases with increase in Reynolds number Re. It is observed from Figure 4 that the primary velocity $u_{1}$ decreases while the magnitude of secondary velocity $w_{1}$ increases with increase in Hall parameter $\beta_{e}$. Figure 5 displays that both the primary velocity $u_{1}$ and the magnitude of secondary velocity $w_{1}$ decrease with increase in ion-slip parameter $\beta_{i}$. It is also seen from Figure 6 that the primary velocity $u_{1}$ increases whereas the magnitude of secondary velocity $w_{1}$ decreases with increase in time $\tau$.

$$
u_{1}+i w_{1}=1-e^{-\frac{\mathrm{Re}}{2} \eta}\left[\cosh \left(\alpha_{1}-i \beta_{1}\right) \eta-\frac{\cosh \left(\alpha_{1}-i \beta_{1}\right)}{\sinh \left(\alpha_{1}-i \beta_{1}\right)} \sinh \left(\alpha_{1}-i \beta_{1}\right) \eta+\sum_{n=1}^{\infty} \frac{2 n \pi(-1)^{n}}{n^{2} \pi^{2}+\left(\alpha_{1}-i \beta_{1}\right)^{2}} e^{s_{1} \tau} \sin n \pi(1-\eta)\right]
$$




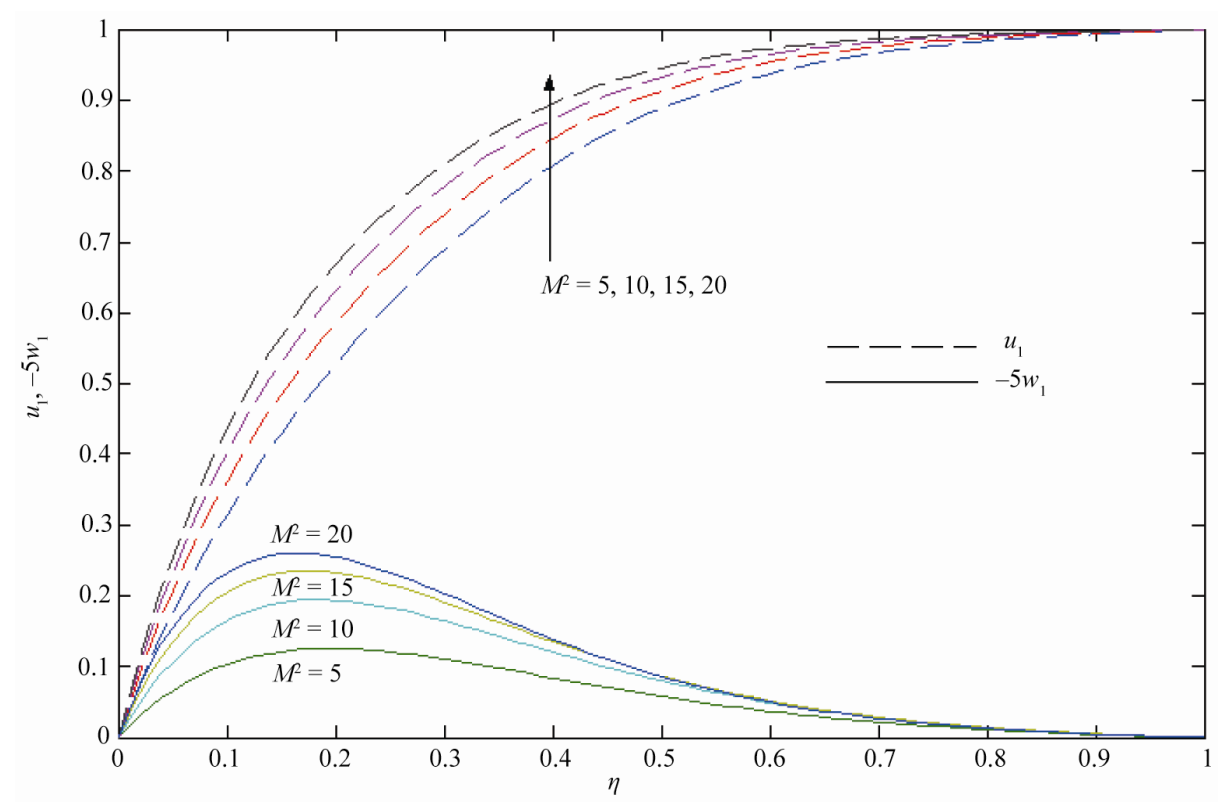

Figure 2. Velocity profiles for $M^{2}$ when $\operatorname{Re}=5, \beta_{e}=0.5, \beta_{i}=0.5$ and $\tau=0.02$.

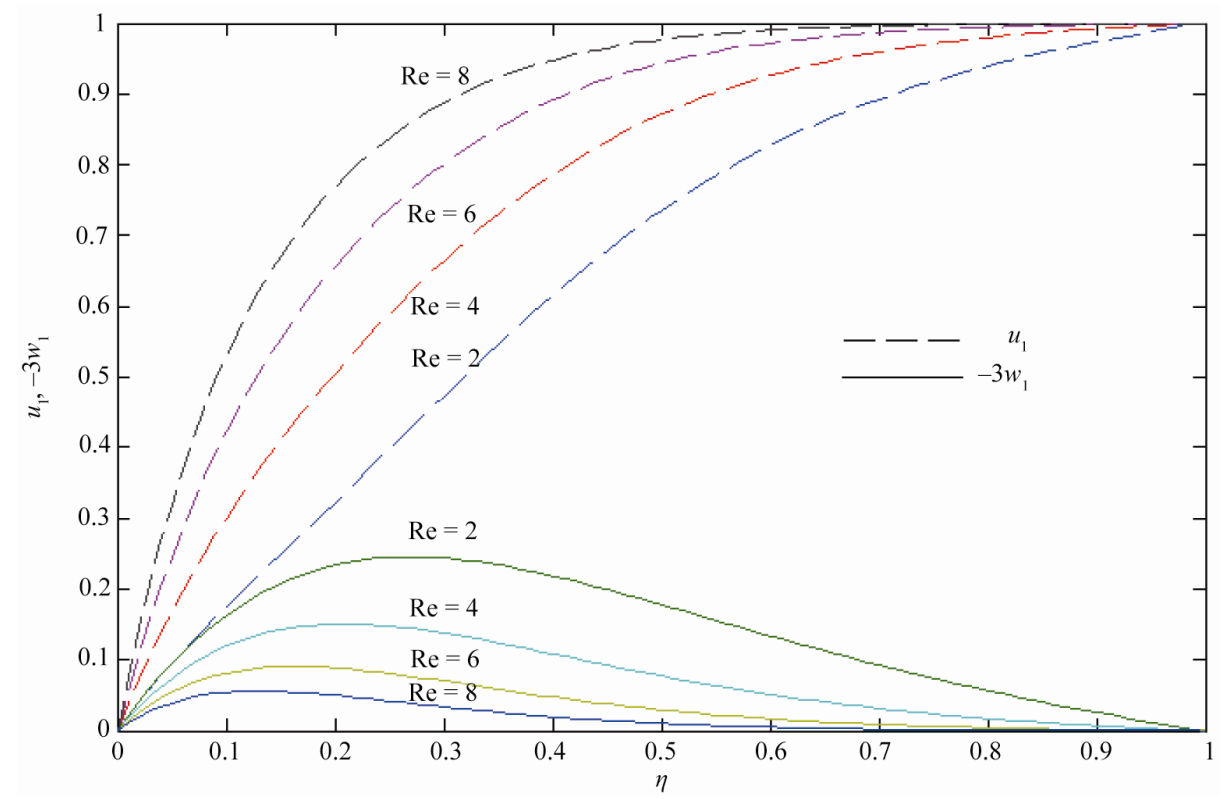

Figure 3. Velocity profiles for Re when $M^{2}=5, \beta_{e}=0.5, \beta_{i}=0.5$ and $\tau=0.02$.

For small values of time, we have drawn the velocity components $u_{1}$ and $w_{1}$ on using the exact solution given by Equation (25) and the series solution given by Equations (30) and (31) in Figures 7, 8. It is seen that the series solution given by (30) and (31) converge more quickly than the exact solution given by (25) for small time. Hence we conclude that for small time, the numerical values of the velocities can be calculated from the Equations (30) and (31) instead of Equation (25).

The non-dimensional shear stresses due to the primary and the secondary velocities at the stationary plate

$$
\begin{aligned}
& \begin{aligned}
\eta=0 & \text { are given by } \\
\tau_{x_{0}}=\left[\frac{\mathrm{d} u_{1}}{\mathrm{~d} \eta}\right]_{\eta=0} & =\frac{1}{2} \operatorname{Re}+\frac{2\left(\alpha_{1} \sinh 2 \alpha_{1}+\beta_{1} \sin 2 \beta_{1}\right)}{\cosh 2 \alpha_{1}-\cos 2 \beta_{1}} \\
& +\sum_{n=1}^{\infty} \frac{e^{-\left(n^{2} \pi^{2}+\frac{\mathrm{Re}^{2}}{4}+\alpha\right) \tau}}{\left(n^{2} \pi^{2}+\frac{\mathrm{Re}^{2}}{4}+\alpha\right)^{2}+\beta^{2}} \\
& \times\left[\left(n^{2} \pi^{2}+\frac{\mathrm{Re}^{2}}{4}+\alpha\right) \cos \beta \tau+\beta \sin \beta \tau\right],
\end{aligned}
\end{aligned}
$$




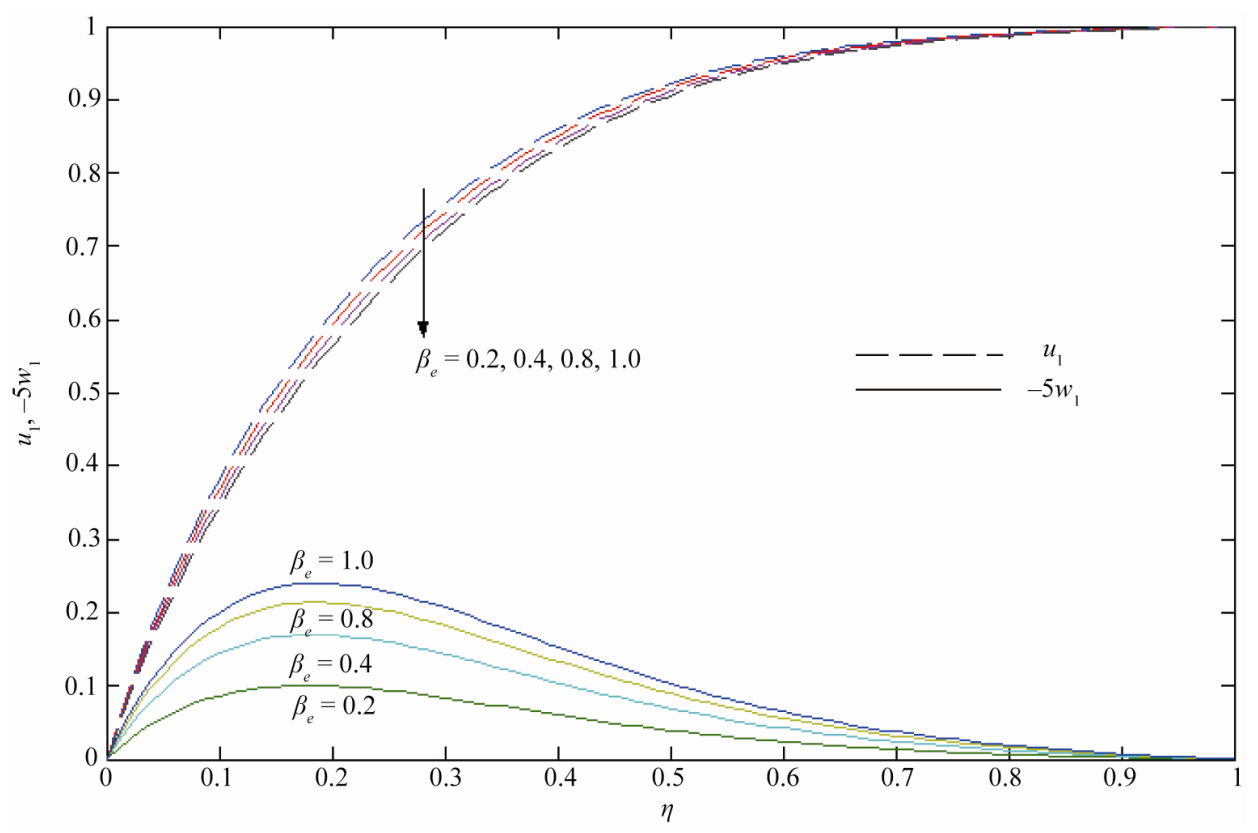

Figure 4. Velocity profiles for $\beta_{e}$ when $M^{2}=5, \operatorname{Re}=5, \beta_{i}=0.5$ and $\tau=0.02$.

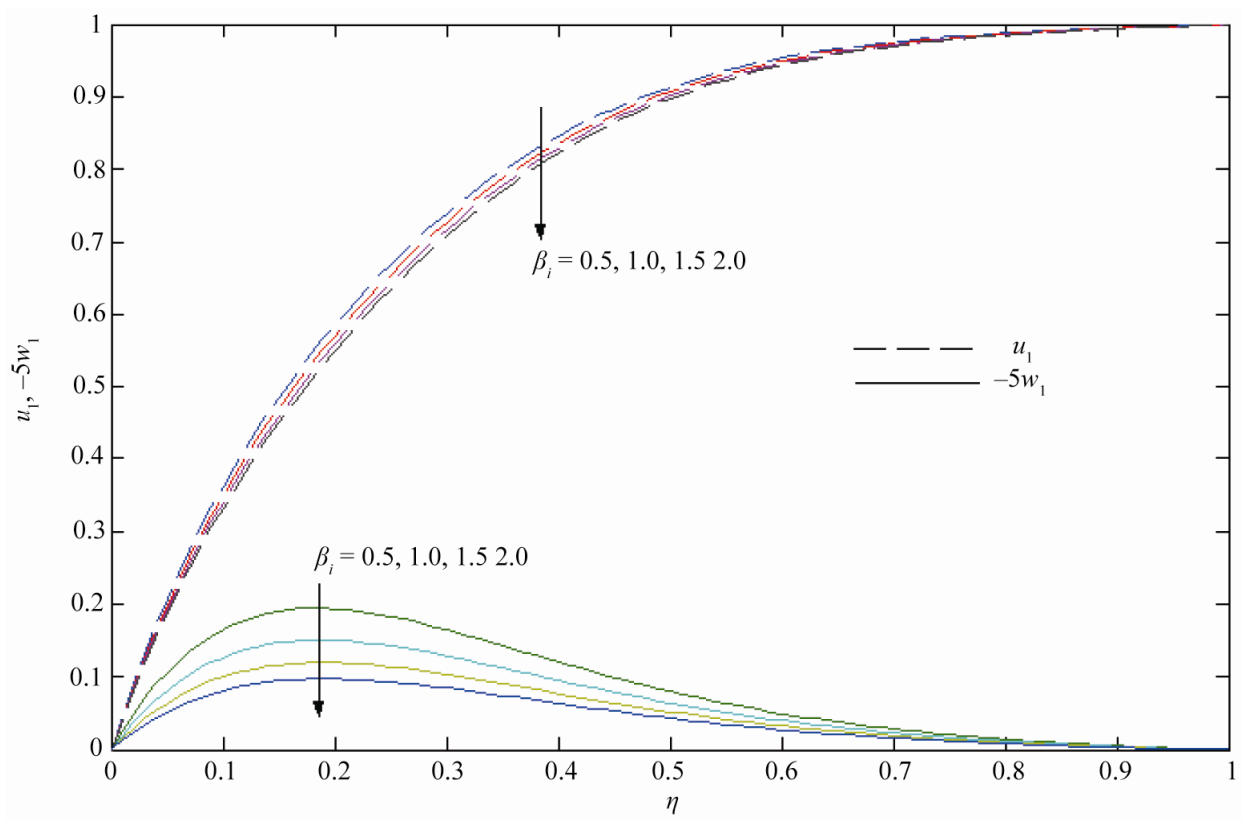

Figure 5. Velocity profiles for $\beta_{i}$ when $M^{2}=5, \operatorname{Re}=5, \beta_{e}=0.5$ and $\tau=0.02$.

$$
\begin{aligned}
\tau_{z_{0}}=\left[\frac{\mathrm{d} w_{1}}{\mathrm{~d} \eta}\right]_{\eta=0} & =\frac{2\left(\alpha_{1} \sinh 2 \alpha_{1}+\beta_{1} \sin 2 \beta_{1}\right)}{\cosh 2 \alpha_{1}-\cos 2 \beta_{1}} \\
& +\sum_{n=1}^{\infty} \frac{e^{-\left(n^{2} \pi^{2}+\frac{\mathrm{Re}^{2}}{4}+\alpha\right) \tau}}{\left(n^{2} \pi^{2}+\frac{\mathrm{Re}^{2}}{4}+\alpha\right)^{2}+\beta^{2}} \\
& \times\left[\beta \cos \beta \tau-\left(n^{2} \pi^{2}+\frac{\mathrm{Re}^{2}}{4}+\alpha\right) \sin \beta \tau\right],
\end{aligned}
$$

where $\alpha, \beta, \alpha_{1}$ and $\beta_{1}$ are given by (26).

Numerical results of the non-dimensional shear stresses $\tau_{x_{0}}$ and $\tau_{z_{0}}$ due to the primary and secondary flows at the plate $\eta=0$ are shown graphically in Figures 9-12 against $M^{2}$ for different values of $\beta_{e}, \beta_{i}$, Re and $\tau$. Figure 9 shows that the shear stresses $\tau_{x_{0}}$ and $\tau_{z_{0}}$ due to the primary and the secondary flows at the stationary plate $\eta=0$ increase with increase in Hall parameter $\beta_{e}$ for fixed value of $M^{2}, \beta_{i}, \tau$ and $\mathrm{Re}$. It is seen from Figure 10 that for fixed value of $M^{2}$, 


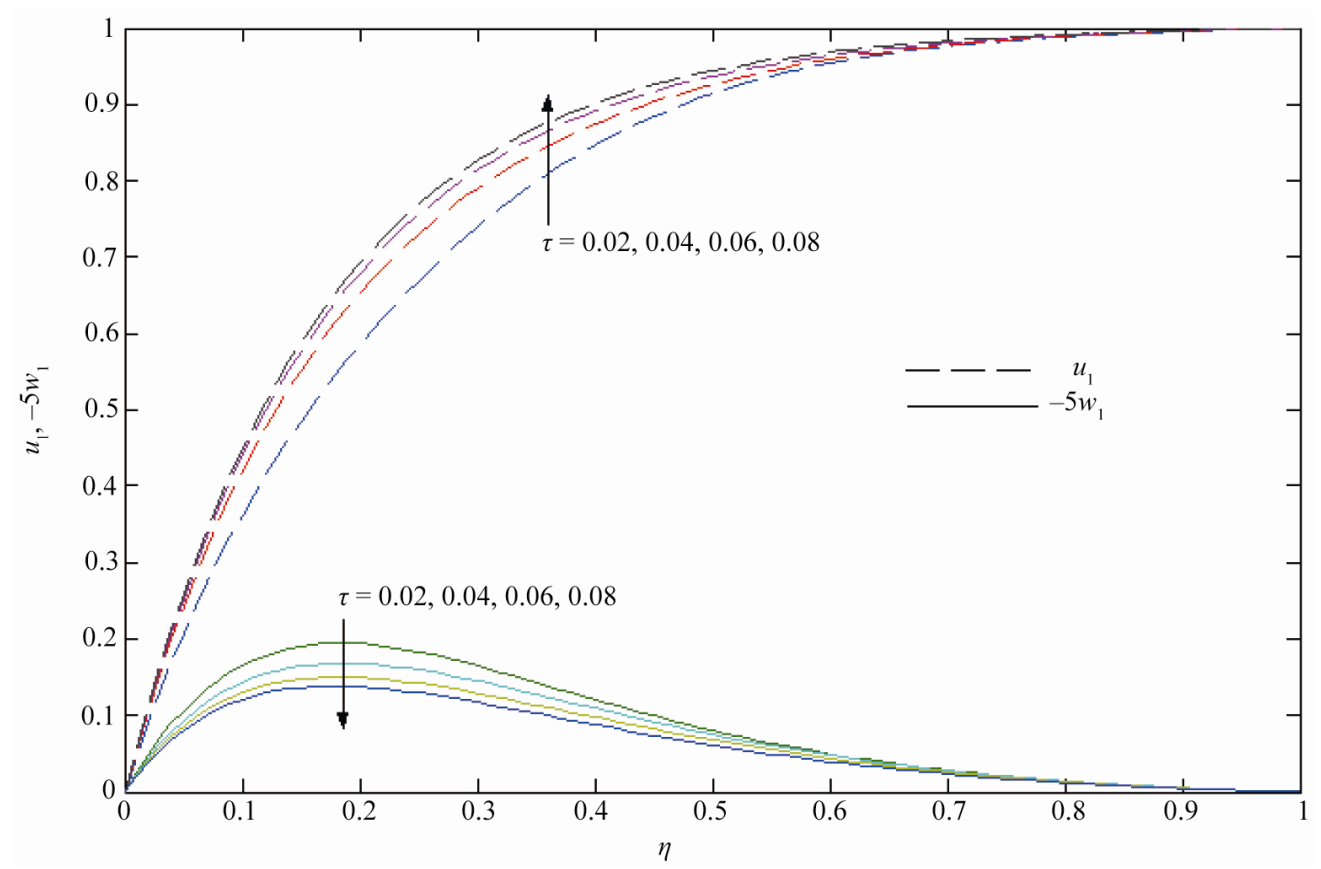

Figure 6. Velocity profiles for time $\tau$ when $M^{2}=5, \operatorname{Re}=5, \beta_{i}=0.5$ and $\beta_{e}=0.5$.

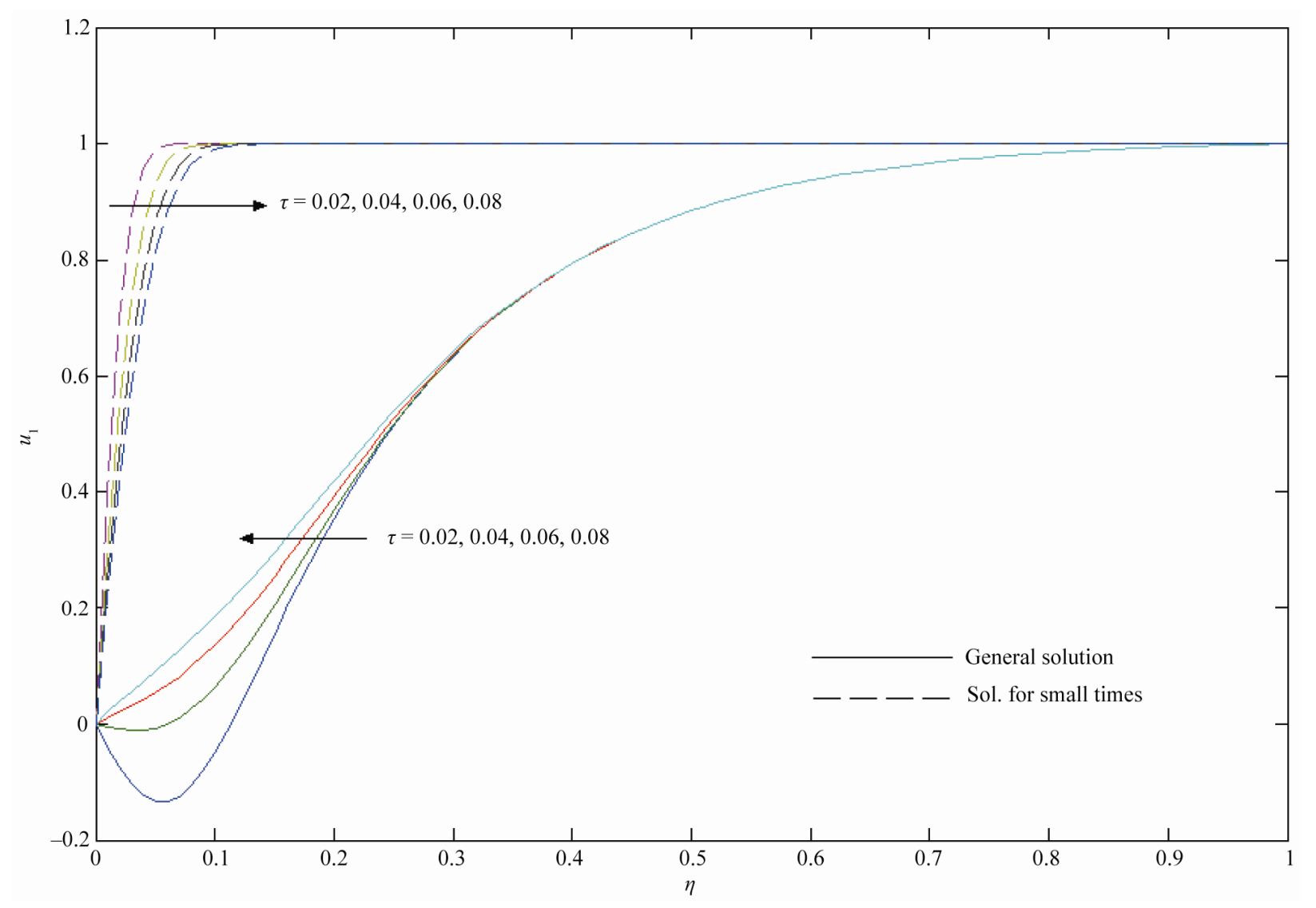

Figure 7. Velocity profiles for general solution and small time solution when $M^{2}=5, \operatorname{Re}=5, \beta_{e}=0.5$ and $\beta_{i}=0.5$.

$\beta_{e}, \tau$ and $\operatorname{Re} . \tau_{x_{0}}$ increases while $\tau_{z_{0}}$ decrease with increase in ion-slip parameter $\beta_{i}$. Figure 11 shows that for fixed value of $M^{2}, \tau_{x_{0}}$ increases while $\tau_{z_{0}}$ decreases with increase in Reynolds number Re. Fur- 


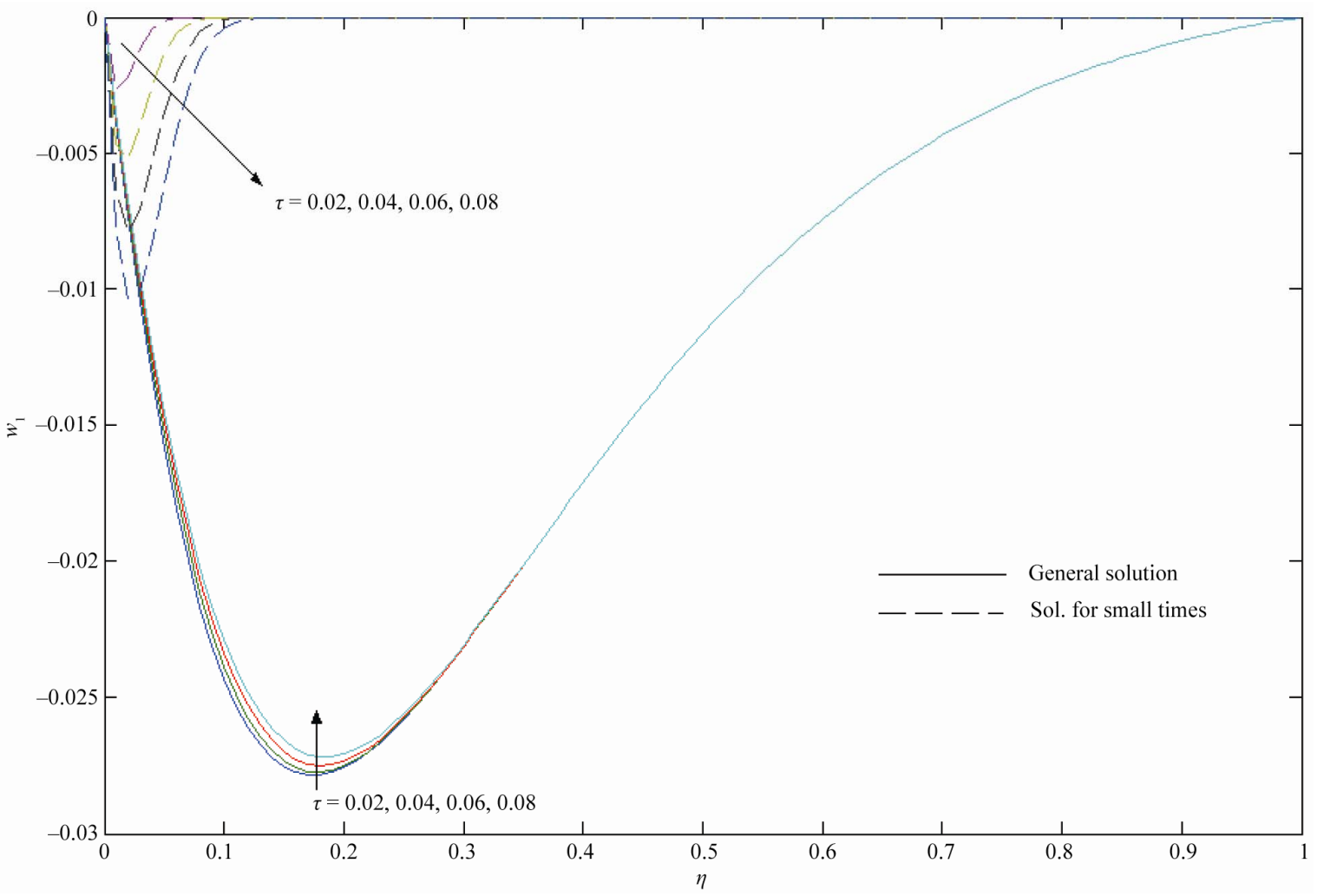

Figure 8. Velocity profiles for general solution and small time solution when $M^{2}=5, \operatorname{Re}=5, \beta_{e}=0.5$ and $\beta_{i}=0.5$.

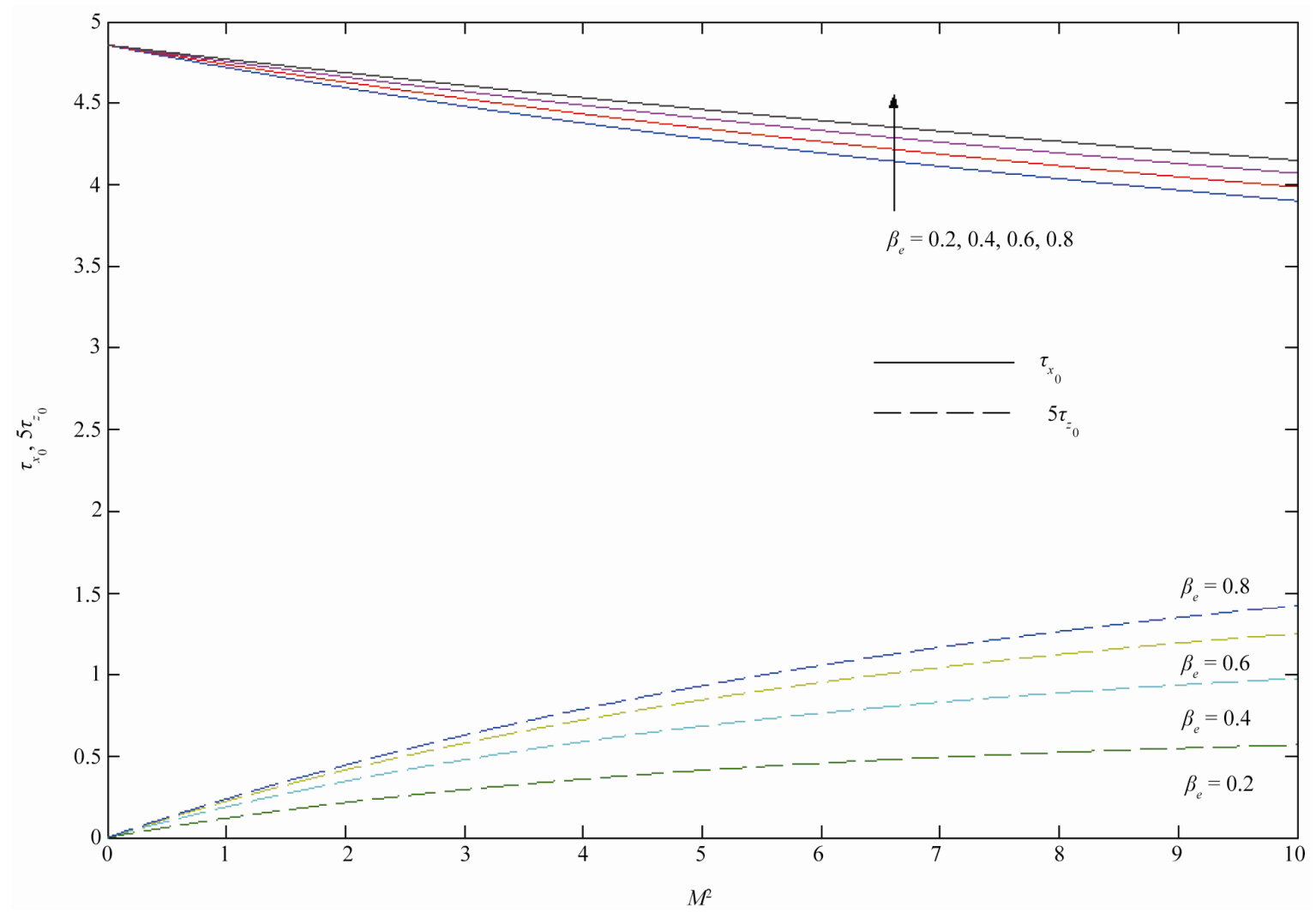

Figure 9. Shear stresses $\tau_{x_{0}}$ and $\tau_{z_{0}}$ for $\beta_{e}$ when $\operatorname{Re}=5, \beta_{i}=0.5$ and $\tau=0.02$. 


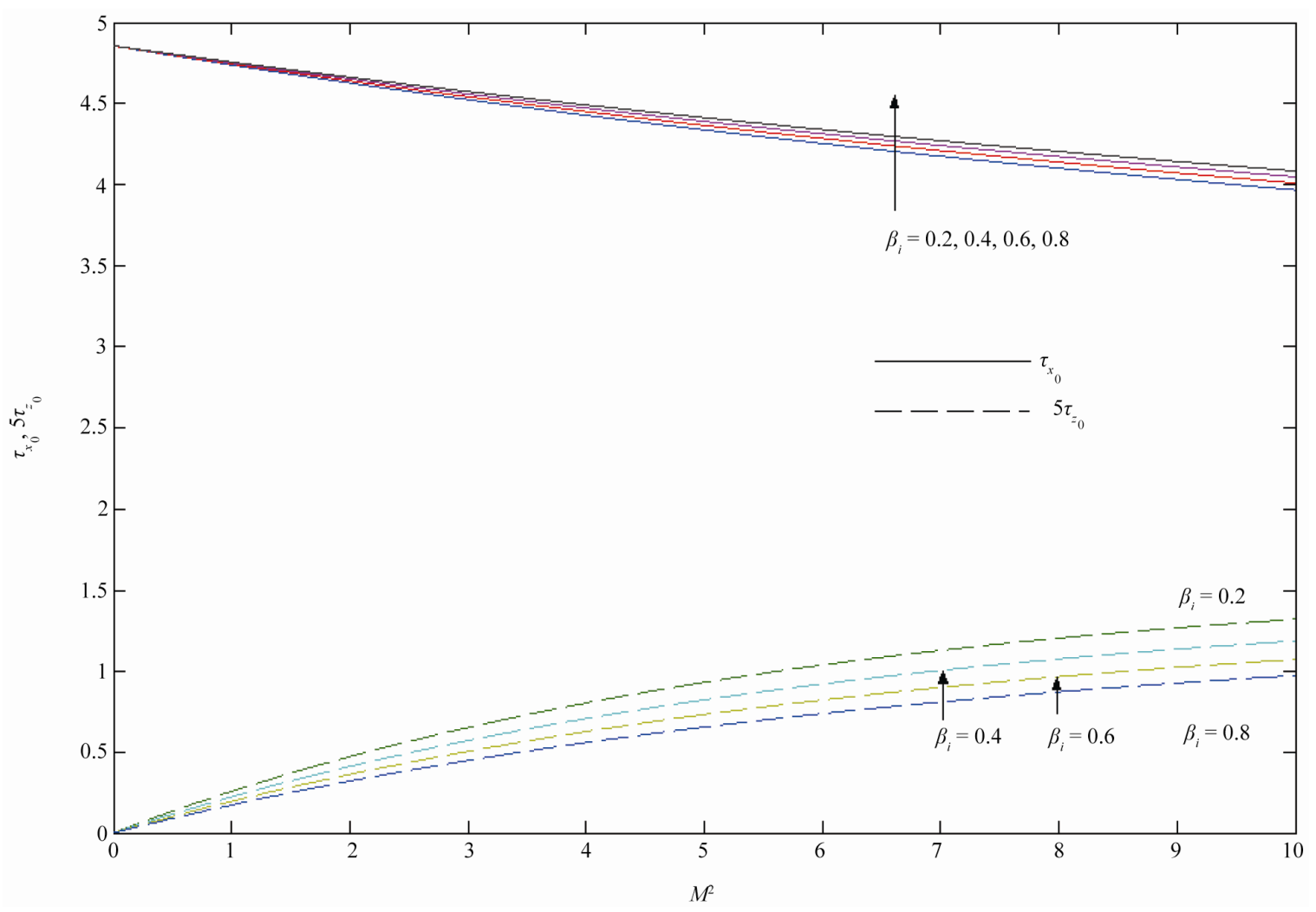

Figure 10. Shear stresses $\tau_{x_{0}}$ and $\tau_{z_{0}}$ for $\beta_{i}$ when $\operatorname{Re}=5, \beta_{e}=0.5$ and $\tau=0.02$.

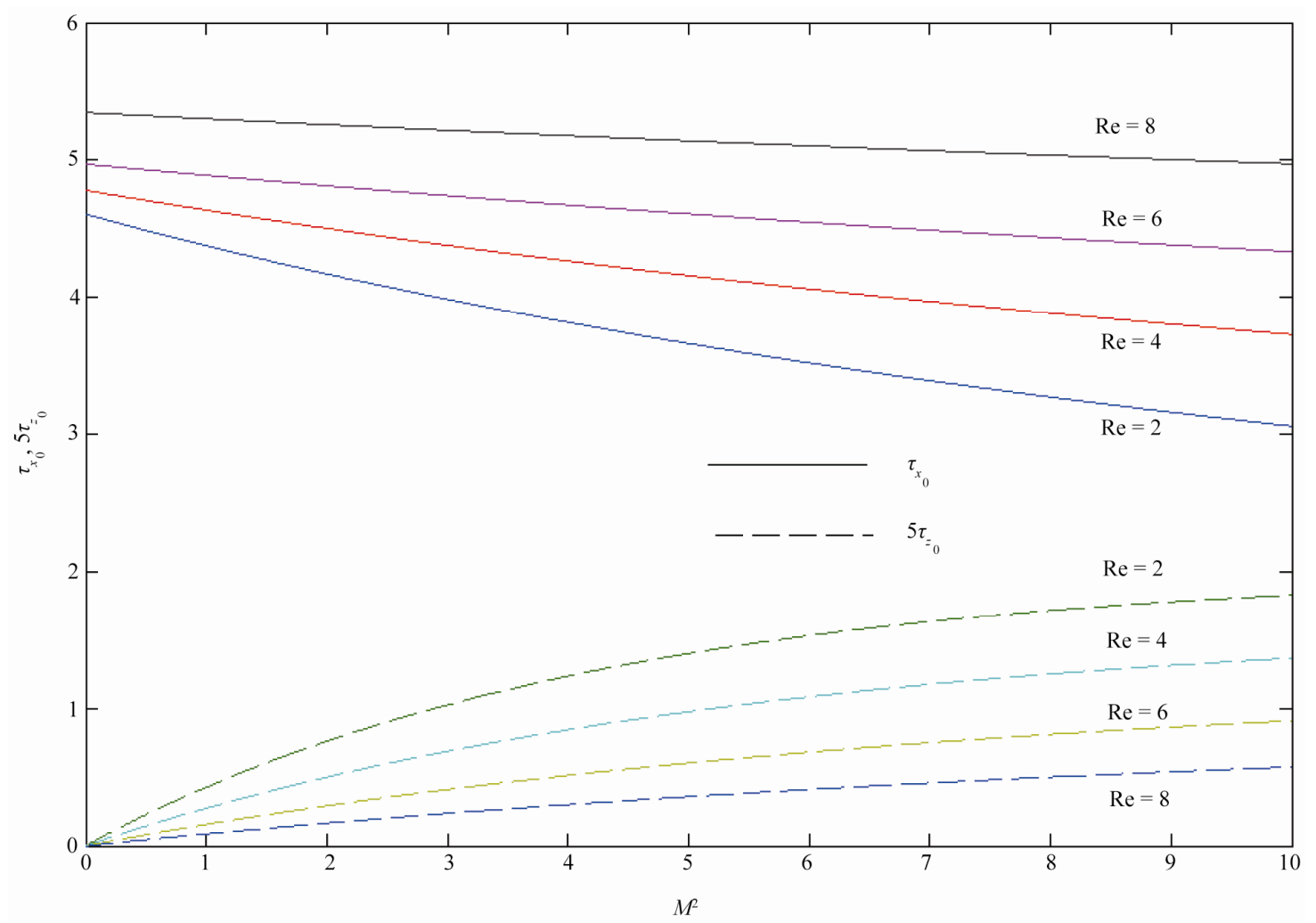

Figure 11. Shear stresses $\tau_{x_{0}}$ and $\tau_{z_{0}}$ for Re when $\beta_{e}=0.5, \beta_{i}=0.5$ and $\tau=0.02$. 


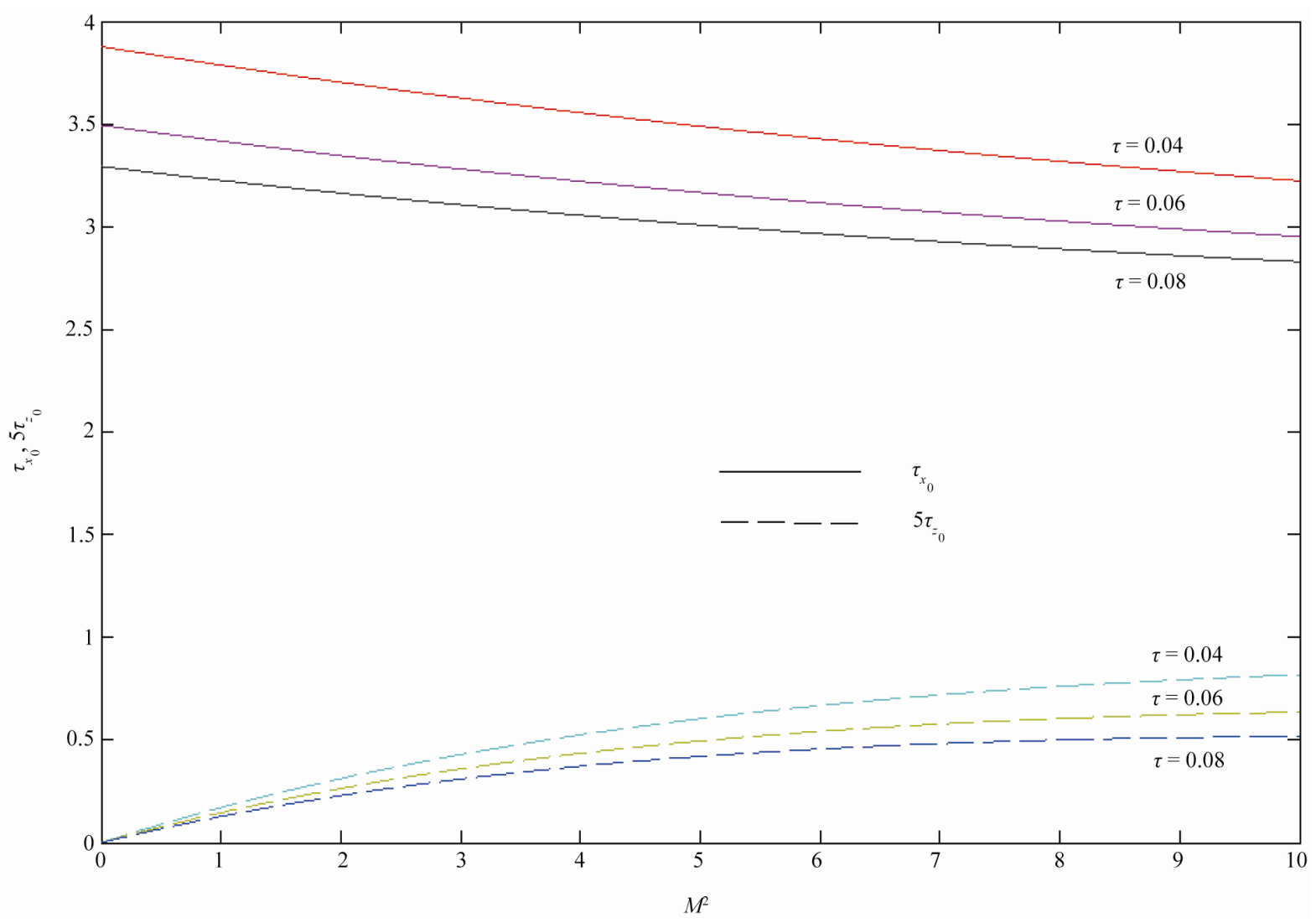

Figure 12. Shear stresses $\tau_{x_{0}}$ and $\tau_{z_{0}}$ for time $\tau$ when $\beta_{e}=0.5, \beta_{i}=0.5$ and $\operatorname{Re}=5$.

ther, it is also found from Figure 12 that both $\tau_{x_{0}}$ and $\tau_{z_{0}}$ decrease with increase in time $\tau$ for fixed value of $M^{2}, \beta_{i}, \beta_{e}$ and $\mathrm{Re}$.

For small times, the shear stress at the plate $\eta=0$ due to the primary and the secondary flows can be obtained as

$$
\begin{aligned}
\tau_{x_{0}} & =\frac{u^{\prime}(0, \tau)}{U} \\
& =\frac{1}{2} e^{-\alpha^{*} \tau}\left[C(0, \tau) \cos \beta^{*} \tau-D(0, \tau) \sin \beta^{*} \tau\right], \\
\tau_{z_{0}} & =\frac{w^{\prime}(0, \tau)}{U} \\
& =\frac{1}{2} e^{-\alpha^{*} \tau}\left[C(0, \tau) \sin \beta^{*} \tau+D(0, \tau) \cos \beta^{*} \tau\right],
\end{aligned}
$$

where

$$
\begin{aligned}
C(\eta, \tau)= & \left(\operatorname{Re} T_{0}+\frac{Y_{-1}}{\sqrt{\tau}}\right)+\alpha^{*}(4 \tau)\left(\operatorname{Re} T_{2}+\frac{Y_{1}}{\sqrt{\tau}}\right) \\
& +\left(\alpha^{* 2}-\beta^{* 2}\right)(4 \tau)^{2}\left(\operatorname{Re} T_{4}+\frac{Y_{3}}{\sqrt{\tau}}\right) \\
& +\left(\alpha^{* 3}-3 \alpha^{*} \beta^{* 2}\right)(4 \tau)^{3}\left(\operatorname{Re} T_{6}+\frac{Y_{5}}{\sqrt{\tau}}\right)+\cdots,
\end{aligned}
$$

$$
\begin{aligned}
D(\eta, \tau)= & \beta^{*}(4 \tau)\left(\operatorname{Re} T_{2}+\frac{Y_{1}}{\sqrt{\tau}}\right) \\
& +2 \alpha^{*} \beta^{*}(4 \tau)^{2}\left(\operatorname{Re} T_{4}+\frac{Y_{3}}{\sqrt{\tau}}\right) \\
& +\left(3 \alpha^{* 2} \beta^{*}-\beta^{* 3}\right)(4 \tau)^{3}\left(\operatorname{Re} T_{6}+\frac{Y_{5}}{\sqrt{\tau}}\right)+\cdots,
\end{aligned}
$$

with

$$
\frac{\mathrm{d} T_{2 n}}{\mathrm{~d} \eta}=-\frac{Y_{2 n-1}}{2 \sqrt{\tau}}
$$

and

$$
\begin{aligned}
& Y_{2 n-1}=\sum_{m=0}^{\infty}\left[i^{2 n-1} \operatorname{erfc}\left(\frac{2 m+2-\eta}{2 \sqrt{\tau}}\right)-i^{2 n-1} \operatorname{erfc}\left(\frac{2 m+\eta}{2 \sqrt{\tau}}\right)\right], \\
& n=0,1,2,3, \cdots
\end{aligned}
$$

For small time, the numerical values of the shear stress components calculated from Equations (34)-(37) are given in Tables 1 and 2 for different values of $\beta_{e}$ and $\tau$. It is observed that for small times the shear stresses calculated from the Equations (36) and (37) give better result than that calculated from Equations (34) and (35). 
Table 1. Shear stresses due to primary flow for $M^{2}=5, S=$ 1.

\begin{tabular}{ccccccc}
\hline \multicolumn{4}{c}{$-\tau_{\times}$(For General solution) } & \multicolumn{3}{c}{$-\tau_{\times}$(Solution for small times) } \\
\hline$\beta_{e} / \tau$ & 0.005 & 0.010 & 0.015 & 0.005 & 0.010 & 0.015 \\
0.0 & 5.896093 & 3.644242 & 2.673381 & 5.896093 & 3.644238 & 2.673358 \\
0.5 & 6.032860 & 3.765285 & 2.782767 & 6.032872 & 3.765358 & 2.782959 \\
1.0 & 6.290388 & 3.998794 & 2.998242 & 6.290404 & 3.998886 & 2.998493 \\
1.5 & 6.515985 & 4.208713 & 3.196242 & 6.515995 & 4.208762 & 3.196378 \\
\hline
\end{tabular}

Table 2. Shear stresses due to secondary flow for $M^{2}=5, S$ $=1$.

\begin{tabular}{ccccccc}
\hline \multicolumn{6}{c}{$\tau_{y}$ (For General Solution) } & \multicolumn{2}{c}{$\tau_{y}$ (Solution for small times) } \\
$\beta_{e} / \tau$ & 0.005 & 0.010 & 0.015 & 0.005 & 0.010 & 0.015 \\
0.0 & 0.000000 & 0.000000 & 0.000000 & 0.000000 & 0.000000 & 0.000000 \\
0.5 & 0.393570 & 0.361540 & 0.337486 & 0.393615 & 0.361790 & 0.338174 \\
1.0 & 0.595888 & 0.555407 & 0.524776 & 0.595908 & 0.555517 & 0.525087 \\
1.5 & 0.660252 & 0.622620 & 0.594005 & 0.660258 & 0.622650 & 0.594089 \\
\hline
\end{tabular}

\section{Steady state solution}

The steady state velocity components are obtained from (25) by letting $\tau \rightarrow \infty$ as (see Equation (42))

Now, we shall discuss the following cases:

Case 1): When $M^{2} \gg 1$ and $\operatorname{Re} \ll 1$.

In this case, Equation (42) gives

$$
\begin{gathered}
u_{1}=1-e^{-\left(\frac{\mathrm{Re}}{2}+\alpha_{1}\right) \eta} \cos \beta_{1} \eta \\
w_{1}=-e^{-\left(\frac{\mathrm{Re}}{2}+\alpha_{1}\right) \eta} \sin \beta_{1} \eta
\end{gathered}
$$

where

$$
\begin{aligned}
& \alpha_{1}=M\left\{\frac{1+\beta_{e} \beta_{i}}{\left(1+\beta_{e} \beta_{i}\right)^{2}+\beta_{e}^{2}}\right\}^{\frac{1}{2}}, \\
& \beta_{1}=\frac{M \beta_{e}}{\left[\left\{\left(1+\beta_{e} \beta_{i}\right)^{2}+\beta_{e}^{2}\right\}\left(1+\beta_{e} \beta_{i}\right)\right]^{\frac{1}{2}}} .
\end{aligned}
$$

It is seen from Equations (43) and (44) that there exists a single-deck boundary layer of thickness of the order

$\mathrm{O}\left(\frac{\mathrm{Re}}{2}+\alpha_{1}\right)^{-1}$ where $\alpha_{1}$ is given by (45). It is seen that the thickness of this boundary layer increases with increase in either Hall parameter $\beta_{e}$ or ion-slip parameter $\beta_{i}$ but it decreases with increase in either Hartmann number $M$ or Reynolds number $\mathrm{Re}$.

Case 2): When $\operatorname{Re} \gg 1, M^{2} \ll 1$.
In this case, the velocity distributions given by (42) become

$$
\begin{aligned}
& u_{1}=1-e^{-\alpha_{1} \eta} \cos \beta_{1} \eta \\
& w_{1}=e^{-\alpha_{1} \eta} \sin \beta_{1} \eta,
\end{aligned}
$$

where

$$
\begin{aligned}
& \alpha_{1}=\operatorname{Re}\left\{1+\frac{M^{2}}{\operatorname{Re}^{2}} \frac{\left(1+\beta_{e} \beta_{i}\right)}{\left(1+\beta_{e} \beta_{i}\right)^{2}+\beta_{e}^{2}}\right\}, \\
& \beta_{1}=\frac{M^{2} \beta_{e}}{\operatorname{Re}^{2}\left\{\left(1+\beta_{e} \beta_{i}\right)^{2}+\beta_{e}^{2}\right\}} .
\end{aligned}
$$

Equations (46) and (47) show the existence of singledeck boundary layer of thickness of order $\mathrm{O}\left(\alpha_{1}^{-1}\right)$ where $\alpha_{1}$ is given by (48). The thickness of this layer increases with increase in either Hall parameter $\beta_{e}$ or ion-slip parameter $\beta_{i}$ as $\alpha_{1}$ decreases with increase in either $\beta_{e}$ or $\beta_{i}$.

\section{Single Plate Motion}

As $h \rightarrow \infty$, the velocity distribution given by (42) becomes

$$
\begin{aligned}
& u_{1}=1-e^{-\left(\frac{s}{2}+\alpha_{1}\right) \eta} \cos \beta_{1} \eta \\
& w_{1}=e^{-\left(\frac{s}{2}+\alpha_{1}\right) \eta} \sin \beta_{1} \eta
\end{aligned}
$$

where

$$
\begin{aligned}
& \alpha_{1}=\frac{1}{\sqrt{2}}\left[\left\{\left(\frac{S^{2}}{4}+\alpha\right)^{2}+\beta^{2}\right\}^{\frac{1}{2}}+\left(\frac{S^{2}}{4}+\alpha\right)\right]^{\frac{1}{2}}, \\
& \beta_{1}=\frac{1}{\sqrt{2}}\left[\left\{\left(\frac{S^{2}}{4}+\alpha\right)^{2}+\beta^{2}\right\}^{\frac{1}{2}}-\left(\frac{S^{2}}{4}+\alpha\right)\right]^{\frac{1}{2}} \\
& \eta=\frac{U z}{v}, \quad S=\frac{v_{0}}{U}, \quad M^{2}=\frac{\sigma B_{0}^{2} v}{\rho U^{2}}
\end{aligned}
$$

and $\alpha, \beta$ are given by (26). It is clear from above Equations (49) and (50) that the flow exhibits a boundary layer behavior with boundary layer thickness of order of $\mathrm{O}\left(\frac{S}{2}+\alpha_{1}\right)^{-1}$. Since $\alpha_{1}$ increases with increase in either $S$ or $M^{2}$ it means that increase in either suction pa-

$$
u_{1}+i w_{1}=1-\left[\frac{\cosh \left(\alpha_{1}-i \beta\right)}{\sinh \left(\alpha_{1}-i \beta_{1}\right)} \sinh \left(\alpha_{1}-i \beta_{1}\right) \eta-\cosh \left(\alpha_{1}-i \beta_{1}\right) \eta\right] e^{-\frac{\mathrm{Re}}{2} \eta}
$$


rameter $S$ or magnetic parameter $M^{2}$ causes thinning of the boundary layer. Further, for fixed $S$ and $M^{2}$, $\alpha_{1}$ decreases with increase in either Hall parameter $\beta_{e}$ or ion-slip parameter $\beta_{i}$. Hence, we conclude that the boundary layer thickness near the plate $\eta=0$ increases with increase in either $\beta_{e}$ or $\beta_{i}$. The solutions given by (49) and (50) are also valid for the blowing $(S<0)$ at the plate.

In the absence of ion-slip $\left(\beta_{i}=0\right)$, the above Equations (49) and (50) become

$$
\begin{gathered}
u_{1}=1-e^{-\left(\frac{s}{2}+\alpha\right) \eta} \cos \beta \eta, \\
w_{1}=e^{-\left(\frac{s}{2}+\alpha\right) \eta} \sin \beta \eta,
\end{gathered}
$$

where

$$
\begin{gathered}
\alpha=\frac{1}{\sqrt{2}}\left[\left\{\left(\frac{S^{2}}{4}+\frac{M^{2}}{1+\beta_{e}^{2}}\right)^{2}+\left(\frac{\beta_{e} M^{2}}{1+\beta_{e}^{2}}\right)^{2}\right\}^{\frac{1}{2}}\right. \\
\left.+\left(\frac{S^{2}}{4}+\frac{M^{2}}{1+\beta_{e}^{2}}\right)\right]^{\frac{1}{2}}, \\
\beta=\frac{1}{\sqrt{2}}\left[\left\{\left(\frac{S^{2}}{4}+\frac{M^{2}}{1+\beta_{e}^{2}}\right)^{2}+\left(\frac{\beta_{e} M^{2}}{1+\beta_{e}^{2}}\right)^{2}\right\}^{\frac{1}{2}}\right. \\
\left.-\left(\frac{S^{2}}{4}+\frac{M^{2}}{1+\beta_{e}^{2}}\right)\right]^{\frac{1}{2}} .
\end{gathered}
$$

Equations (52) and (53) coincide with Equations (36) and (37) of Gupta [16] when $\beta_{i}=0$ (absence of ionslip).

\section{Conclusion}

Combined effects of Hall current and ion-slip on the unsteady MHD Couette flow between two infinite horizontal parallel porous plates under the boundary layer approximations have been studied. It is found that the primary velocity $u_{1}$ decreases while the magnitude of secondary velocity $w_{1}$ increases with increase in Hall parameter $\beta_{e}$. It is also found that both the primary velocity and the magnitude of secondary velocity decrease with increase in ion-slip parameter $\beta_{i}$. It is observed that a thin boundary layer is formed near the stationary plate for large values of magnetic parameter $M^{2}$ and Reynolds number Re. The thickness of these boundary layers increases with increases in either Hall parameter or ion-slip parameter. Further, it is seen that the shear stresses $\tau_{x_{0}}$ and $\tau_{z_{0}}$ due to the primary and secondary flows at the stationary plate $\eta=0$ increase with increase in Hall parameter $\beta_{e}$ for fixed value of $M^{2}$. It is also seen that for fixed value of $M^{2}, \tau_{x_{0}}$ increases while $\tau_{z_{0}}$ decrease with increase in ion-slip parameter $\beta_{i}$.

\section{REFERENCES}

[1] K. R. Crammer and S.-I. Pai, "Magnetofluid Dynamics for Engineers and Applied Physicists," McGraw-Hill, New York, 1973.

[2] V. M. Soundalgekar, N. V. Vighnesam and H. S. Takhar, "Hall and Ion-Slip Effects in MHD Couette Flow with Heat Transfer," IEEE Transactions on Plasma Science, Vol. 7, No. 3, 1978, pp. 178-182. doi:10.1109/TPS.1979.4317226

[3] H. A. Attia, "Unsteady Couette Flow with Heat Transfer Considering Ion-Slip," Turkish Journal of Physics, Vol. 29, 2005, pp. 379-388.

[4] H. A. Attia, "Unsteady Couette Flow with Heat Transfer of a Viscoelastic Fluid Considering the Ion Slip," Journal of the Korean Physical Society, Vol. 47, No. 5, 2005, pp. 809-817.

[5] H. A. Attia, "Time Varying Hydromagnetic Flow of Dusty Fluid between Parallel Porous Plates Considering the Ion Slip," Journal of Technical Physics, Vol. 47, No. 3, 2006, pp. 131-147.

[6] H. A. Attia, "Analytical Solution for Flow of a Dusty Fluid in a Circular Pipe with Hall and Ion Slip Effects," Chemical Engineering Communications (CEC), Vol. 194, No. 10, 2007, pp. 1287-1296.

[7] H. A. Attia, "Transient Hartmann Flow with Heat Transfer Considering the Ion Slip," Physica Scripta, Vol. 66, 2002, pp. 470-475. doi:10.1238/Physica.Regular.066a00470

[8] M. A. Seddeek, "The Effects of Hall and Ion-Slip Currents on Magneto-Micropolar Fluid and Heat Transfer over a Non-Isothermal Stretching Sheet with Suction and Blowing," Proceedings of the Royal Soceity: London A, Vol. 457, 2001, pp. 3039-3050. doi:10.1098/rspa.2001.0847

[9] P. C. Ram, "The Effects of Hall and Ion-Slip Currents on Free Convective Heat Generating Flow in a Rotating Fluid," International Journal of Energy Research, Vol. 19, No. 5, 1995, pp. 371-376. doi:10.1002/er.4440190502

[10] M. L. Mittal and A. N. Bhat, "Forced Convective Heat Transfer in a MHD Channel with Hall and Ion Slip Currents," Applied Scientific Research, Vol. 35, No. 4, 1979 , pp. 251-264. doi:10.1007/BF00418216

[11] R. N. Jana and N. Datta, "Couette Flow and Heat Transfer in a Rotating System," Acta Meccanica, Vol. 26, No. 1-4, 1977, pp. 301-306. doi:10.1007/BF01177152

[12] A. K. Kanch and R. N. Jana, " Hall Effect on Unsteady Couette Flow under Boundary Layer Approximations," Journal of the Physical Sciences, Vol. 7, 2001, pp. 74-86.

[13] H. A. Attia, "Ion Slip Effect on Unsteady Couette Flow 
with Heat Transfer under Exponential Decaying Pressure Gradient," Tamkang Journal of Science and Engineering, Vol. 12, No. 2, 2009, pp. 209-214.

[14] B.K. Jha and C. A. Apere, "Combined Effect of Hall and Ion-Slip Currents on Unsteady MHD Couette Flows in a Rotating System," Journal of the Physical Society of Japan, Vol. 79, No. 10, 2010, pp. 104401-104401-9.

$$
\text { doi:10.1143/JPSJ.79.104401 }
$$

[15] H. S. Carslaw and J. C. Jaeger, "Conduction of Heat in Solids," Oxford University Press, Oxford, 1959.

[16] A. S. Gupta, "Hydromagnetic Flow Past a Porous Flat Plate with Hall Effects," Acta Mechanica, Vol. 22, No. 3-4, 1975, pp. 281-287. doi:10.1007/BF01170681 